\title{
Características epidemiológicas, clínicas y evolutivas de los pacientes pediátricos con enfermedades crónicas y limitantes, susceptibles de recibir atención por cuidados paliativos en el Hospital Pablo Tobón Uribe, Medellín, Colombia.
}

Fecha de recibido: 31 de enero de 2018 Fecha de aprobación: 26 de abril de 2018
Forma de citar este artículo: Elorza M, García-Salido A, Vanegas C, Fernández M. Características epidemiológicas, clínicas y evolutivas de los pacientes pediátricos con enfermedades crónicas y limitantes, susceptibles de recibir atención por cuidados paliativos en el Hospital Pablo Tobón Uribe, Medellín, Colombia. Med U.P.B. 2018;37(2):116-124. DOI:10.18566/medupb.v37n2.a05

1. Pediatra, intensivista, paliativista, Hospital Pablo Tobón Uribe. Medellín, Colombia.

2. Pediatra, intensivista, paliativista, Hospital Infantil Universitario Niño Jesús de Madrid. Madrid, España.

3. Pediatra, intensivista, epidemiólogo, Hospital Pablo Tobón Uribe y Universidad CES. Medellín, Colombia.

Dirección de correspondencia: Mussatye Elorza Parra. Correo electrónico: mussatye@ hotmail.com
Epidemiological, clinical, and developmental characteristics of pediatric patients with chronic and limiting diseases that were eligible for palliative care at Hospital Pablo Tobon Uribe in Medellín, Colombia / características epidemiológicas, clínicas e evolutivas dos pacientes pediátricos com doenças crônicas e limitantes, susceptíveis de receber atenção por cuidados paliativos no Hospital Pablo Tobón Uribe, Medellín, Colômbia

Mussatye Elorza Parra ${ }^{1}$, Alberto García-Salido ${ }^{2}$, César Vanegas Díaz ${ }^{1}$, Mauricio Fernández Laverde $^{3}$

\section{RESUMEN}

Objetivo: en pediatría, los pacientes susceptibles de recibir cuidados paliativos pueden tener una supervivencia larga pero una vida limitada o amenazada por la enfermedad que padecen, por lo tanto, no solo es importante conocer las causas de muerte sino también la prevalencia de dichas enfermedades. El objetivo de esta investigación fue conocer las características epidemiológicas, clínicas y evolutivas de los niños con enfermedades crónicas y limitantes para la vida, que fueron susceptibles de recibir atención de cuidados paliativos en un hospital pediátrico de alta complejidad, durante los años 2014 y 2015. Metodología: estudio descriptivo y retrospectivo realizado en el Hospital Pablo Tobón Uribe en Medellín, Colombia, en el que se incluyeron 3226 pacientes con enfermedades crónicas y limitantes para la vida. No hubo criterios de exclusión. Se tabularon y analizaron estadísticamente con el paquete estadístico SPSS versión 21. Los datos se obtuvieron de la revisión de las historias clínicas por parte de los investigadores.

Resultados: durante el período de estudio, ingresaron al servicio de pediatría 6230 pacientes. El 52\% (3 226) padecía enfermedades crónicas y limitantes para la vida, y era susceptible de recibir atención de cuidados paliativos. El 58\% de los pacientes era de sexo masculino; $17 \%$ era menor de un año, solo un $2 \%$ representó neonatos. La gran mayoría (48\%) eran mayores de 5 años, con un promedio de edad de 8 años. 21\% tenía diagnóstico oncológico, $20 \%$ tenía malformaciones congénitas y otro $20 \%$ enfermedades hematológicas y del sistema inmune (inmunodeficiencias). Un 22\% restante, con distribución similar, tenía enfermedades metabólicas, afecciones originadas en el período neonatal y enfermedades neurológicas. Solo 3,7\% $(n=119)$ recibió atención de cuidados paliativos. La mortalidad fue de 3 \% (95) para los dos años de estudio. 75\% (71) de los pacientes falleció en la UCIP, 63\% (60) tenía ventilación mecánica invasiva. 
Conclusiones: los cuidados paliativos deben desarrollarse y ser accesibles para todos los niños con enfermedades que amenazan su vida con el objetivo de mejorar el manejo de síntomas y su calidad de vida.

Palabras clave: cuidados paliativos; cuidado terminal; niño; enfermedad crónica.

\section{ABSTRACT}

Objective: In pediatrics, patients that are susceptible to palliative care may have a long survival, but a life that is limited or threatened by their disease. Therefore, it is not only important to know the causes of death, but also the prevalence of such diseases. The aim of this research was to determine the epidemiological, clinical, and evolutionary characteristics of children with chronic and life-limiting diseases who received palliative care in a highly complex pediatric hospital during 2014 and 2015.

Methodology: descriptive, retrospective study, carried out at Hospital Pablo Tobon Uribe in Medellin, Colombia, in which 3226 patients with chronic and life-limiting diseases were included. There were no exclusion criteria. Data were tabulated and analyzed using SPSS version 21 statistical package. Data were obtained by the researchers' review of the clinical records.

Results: During the study period, 6230 patients were admitted to the pediatric ward, $52 \%$ (3 226) of whom had chronic and life-limiting diseases that could receive palliative care. $58 \%$ of the patients were male, $17 \%$ were under one year, and only $2 \%$ were neonates. The majority (48\%) were older than 5 years, with an average age of 8 years. $21 \%$ had cancer diagnosis, $20 \%$ had congenital malformations, and another $20 \%$ had hematological and immune system diseases (immunodeficiency). Another $22 \%$, with similar distribution, had metabolic diseases, conditions originated in the neonatal period, and neurological diseases. Only 3.7\% $(n=119)$ received palliative care. Mortality was 3\% (95) for the duration of the study ( 2 years). $75 \%$ (71) of the patients died in the PICU; $63 \%$ (60) had invasive mechanical ventilation.

Conclusions: Palliative care should be developed and accessible to all children with lifethreatening illnesses to improve symptom management and quality of life.

Keywords: palliative care; terminal care; child; chronic disease.

\section{RESUMO}

Objetivo: em pediatria, os pacientes susceptíveis de receber cuidados paliativos podem ter uma supervivência longa mas uma vida limitada ou ameaçada pela doença que padecem, por tanto, não só é importante conhecer as causas de morte senão também a prevalência de ditas doenças. O objetivo desta investigação foi conhecer as características epidemiológicas, clínicas e evolutivas das crianças com doenças crônicas e limitantes para a vida, que foram susceptíveis de receber atenção de cuidados paliativos num hospital pediátrico de alta complexidade, durante os anos 2014 e 2015.

Metodologia: estudo descritivo e retrospectivo realizado no Hospital Pablo Tobón Uribe em Medellín, Colômbia, no qual se incluíram 3226 pacientes com doenças crônicas e limitantes para a vida. Não houve critérios de exclusão. Se tabelaram e analisaram estatisticamente com o pacote estatístico SPSS versão 21. Os dados se obtiveram da revisão das histórias clínicas por parte dos pesquisadores.

Resultados: durante o período de estudo, ingressaram ao serviço de pediatria 6230 pacientes. 52\% (3 226) padecia doenças crônicas e limitantes para a vida, e era susceptível de receber atenção de cuidados paliativos. $58 \%$ dos pacientes era do sexo masculino; $17 \%$ era menor de um ano, só um $2 \%$ representou neonatos. A grande maioria (48\%) eram maiores de 5 anos, com uma média de idade de 8 anos. 21\% tinha diagnóstico oncológico, $20 \%$ tinha malformações congénitas e outro $20 \%$ doenças hematológicas e do sistema imune (imunodeficiências). Um 22\% restante, com distribuição similar, tinha doenças metabólicas, afecções originadas no período neonatal e doenças neurológicas. Só $3,7 \%(n=119)$ recebeu atenção de cuidados paliativos. A mortalidade foi de 3 
\% (95) para os dois anos de estudo. 75\% (71) dos pacientes faleceu na UCIP, 63\% (60) tinha ventilação mecânica invasiva.

Conclusões: os cuidados paliativos devem desenvolver-se e ser acessíveis para todas as crianças com doenças que ameaçam sua vida com o objetivo de melhorar o manejo de sintomas e sua qualidade de vida.

Palavras chave: cuidados paliativos; cuidado terminal; criança; doença crônica.

\section{INTRODUCCIÓN}

Según la Organización Mundial de la Salud, los cuidados paliativos pediátricos "comienzan cuando se diagnostica una enfermedad amenazante para la vida y continúan independientemente de si el niño recibe o no tratamiento de la propia enfermedad"1.

En Colombia, según los últimos datos de mortalidad actualizados del Departamento Administrativo Nacional de Estadística, en 2013 murieron 15675 menores de 18 años. De estas muertes, más de la mitad (8 468), fue atribuible a una causa previsible ${ }^{2}$. Las causas previsibles de muerte son aquellos diagnósticos de enfermedades que amenazan o limitan la vida de un paciente ocasionando un deterioro de su calidad de vida y que se consideran susceptibles de entrar en un programa de cuidados paliativos.

Actualmente, no existe un listado oficial de estas enfermedades. Sin embargo, el informe "Palliative Care Statistics for Children and Young Adults" publicado en el 2007 por el Departamento de Salud de Londres, agrupa estos diagnósticos según los códigos de la Clasificación Internacional de Enfermedades (CIE-10), lo cual es útil para estudios de mortalidad y prevalencia ${ }^{3}$.

Se estima que la tasa de prevalencia para niños y jóvenes que puedan requerir cuidados paliativos es de $10-16$ por cada 10000 habitantes menores de 19 años (15 por cada 10000 si se excluyen las muertes neonatales). De ellos, $50 \%$ puede llegar a necesitar la atención especializada de una unidad de cuidados paliativos pediátricos ${ }^{4,5}$.

El Hospital Pablo Tobón Uribe es una institución de cuarto nivel de atención y de referencia en todo el país. El área de pediatría atiende aproximadamente 3500 niños menores de 16 años anualmente, de los cuales se desconoce cuál es la prevalencia de pacientes con enfermedades crónicas y limitantes para la vida que son susceptibles de recibir atención paliativa. Esto ha limitado la derivación oportuna de los pacientes y revela la falta de conocimiento acerca del cuidado integral de estos niños.

Con este estudio nos propusimos conocer las características epidemiológicas, clínicas y evolutivas de los niños con enfermedades crónicas y limitantes para la vida, que fueron susceptibles de recibir atención de cuidados paliativos en el Hospital Pablo Tobón Uribe (HPTU) en los 2 últimos años.

\section{METODOLOGÍA}

Se llevó a cabo un estudio de tipo descriptivo, retrospectivo, en el que se incluyeron pacientes menores de 16 años con diagnóstico de enfermedades limitantes o amenazantes para la vida, que ingresaron al servicio de hospitalización de pediatría y cuidado crítico del HPTU, durante los años 2014 y 2015. No hubo criterios de exclusión.

Los datos se obtuvieron a través del sistema de gestión de información clínica del HPTU. La información recolectada se tabuló en el programa Microsoft Office Excel 2015 y se exportó al programa estadístico SPSS versión 21.0.

Las variables de diagnósticos en códigos de la CIE-10 de enfermedades amenazantes y limitantes para la vida fueron extraídas del informe "Palliative Care Statistics for Children and Young Adults" publicado en el 2007 por el Departamento de Salud de Londres. 


\section{RESULTADOS}

\section{Características de la población}

Durante el período de estudio, comprendido entre el $1^{\circ}$ de enero de 2014 y el 31 de diciembre de 2015, ingresaron al servicio de pediatría del Hospital Pablo Tobón Uribe de Medellín 6230 pacientes, de estos, 52\% (3 226 niños) padecía enfermedades crónicas y limitantes para la vida y era susceptible de recibir atención de cuidados paliativos.

De la muestra, $58 \%$ de los pacientes era del sexo masculino. El 17\% era menor de un año, solo un 2\% era neonatos. La gran mayoría (48\%) eran mayores de 5 años, con un promedio de edad de 8 años y rango entre 1 día y 15 años. En la Tabla 1 pueden verse las características sociodemográficas de los pacientes.

El 71\% procedía del Valle de Aburrá y el 15\% de las otras de regiones de Antioquia. El 14\% restante, provenía de otros departamentos de Colombia. En la Tabla 2 puede verse la distancia entre la residencia de los pacientes hasta el HPTU.

\section{Diagnósticos, promedio de estancia y atención por cuidados paliativos}

Considerando el total de niños incluidos con enfermedades crónicas y limitantes para la vida que fueron susceptibles de recibir atención de cuidados paliativos, $21 \%$ de ellos tenía diagnóstico oncológico, 20\% tenía malformaciones congénitas y otro $20 \%$, enfermedades hematológicas y del sistema inmune (inmunodeficiencias). Un $22 \%$ restante, con distribución similar, tenía enfermedades metabólicas, afecciones originadas en el período neonatal y enfermedades neurológicas. En la Tabla 3 se presentan los diagnósticos de los pacientes.

De los 3226 niños, solo el 3.7\% ( $n=119)$ recibió atención de cuidados paliativos. La mitad de las referencias a este servicio fueron durante los reingresos del paciente $y$ la otra mitad, en el primer ingreso hospitalario.

El promedio de estancia fue de 11 días, un rango entre 1 y 342 días, con una desviación estándar de 19,6. El $34 \%$ de los pacientes $(n=1113)$ tuvo varios ingresos a hospitalización durante el período de estudio.

Tabla 1. Características demográficas de los niños con enfermedades crónicas y limitantes para la vida, susceptibles de recibir atención de cuidados paliativos en el Hospital Pablo Tobón Uribe en Medellín, Colombia, entre el $1^{\circ}$ de enero de 2014 y el 31 de diciembre de 2015.

\begin{tabular}{lcc}
\hline Variable Sexo & $\mathbf{n}$ & $\%$ \\
\hline Masculino & 1878 & 58 \\
Femenino & 1348 & 42 \\
Total & 326 & 100 \\
Variable Edad & $\mathrm{n}$ & $\%$ \\
Menor de 1 mes & 65 & 2.0 \\
1 mes -12 meses & 479 & 15.0 \\
1 año - 5 años & 1131 & 35.0 \\
Mayor de 5 años & 1551 & 48.0 \\
Total & 3226 & 100.0 \\
Procedencia & $\mathrm{n}$ & $\%$ \\
Valle de Aburrá & 2301 & 71.3 \\
Oriente & 173 & 5.3 \\
Urabá & 89 & 2.7 \\
Suroeste & 68 & 2.1 \\
Norte & 65 & 2.0 \\
Occidente & 49 & 1.5 \\
Nordeste & 337 & 1.3 \\
Bajo Cauca & 326.4 \\
Magdalena medio & 226 & 100 \\
Chocó & 42 & 0.99 \\
Otros departamentos & 32 & 0.55
\end{tabular}


ARTÍCULO ORIGINAL - ORIGINAL ARTICLE - ARTIGO ORIGINAL | Características epidemiológicas, clínicas y evolutivas de los pacientes pediátricos con enfermedades crónicas y limitantes, susceptibles de recibir atención por cuidados paliativos en el Hospital Pablo Tobón Uribe, Medellín, Colombia.

Tabla 2. Distancia en kilómetros desde el sitio de procedencia hasta donde se encuentra el Hospital Pablo Tobó Uribe en Medellín.

\begin{tabular}{lc}
\hline Municipios Valle de Aburrá & Distancia a Medellín en kilómetros \\
\hline Medellín & 0 \\
Bello & 10 \\
Itagüí & 11 \\
Envigado & 10 \\
Caldas & 22 \\
Copacabana & 18 \\
La Estrella & 16 \\
Girardota & 26 \\
Sabaneta & 14 \\
Barbosa & 42 \\
\hline Regiones de Antioquia & Distancia a Medellín en Kilómetros \\
\hline Oriente & 51 \\
Urabá & 373 \\
Suroeste & 160 \\
Norte & 100 \\
Nordeste & 150 \\
Bajo Cauca & 285 \\
Occidente & 115 \\
Magdalena Medio & 185 \\
\hline
\end{tabular}

Tabla 3. Diagnósticos de enfermedades crónicas y limitantes para la vida susceptibles de recibir atención de cuidados paliativos en 3226 niños atendidos entre el $1^{\circ}$ de enero de 2014 y el 31 de diciembre de 2015, en el Hospital Pablo Tobón Uribe en Medellín, Colombia.

\begin{tabular}{lcc}
\hline Diagnóstico & $\mathbf{n}$ & \% del total de pacientes \\
\hline Oncológico & 1112 & 20.6 \\
Malformaciones congénitas & 1071 & 19.8 \\
Enfermedades hematológicas y del sistema inmune & 1054 & 19.5 \\
Enfermedades originadas en el período perinatal & 404 & 7.5 \\
Enfermedades metabólicas & 397 & 7.4 \\
Enfermedades neurológicas & 396 & 7.3 \\
Enfermedades cardiovasculares & 272 & 5 \\
Enfermedades del sistema gastrointestinal & 252 & 4.7 \\
Enfermedades renales & 188 & 3.5 \\
Enfermedades osteomusculares & 116 & 2.1 \\
Enfermedades infecciosas & 89 & 1.6 \\
Trastornos mentales & 32 & 0.6 \\
Enfermedades pulmonares & 7 & 0.1 \\
Otras enfermedades & 9 & 0.2 \\
\hline
\end{tabular}


Mortalidad y características de los pacientes al momento del éxitus

La mortalidad fue del 3\% (n =95) para los dos años de estudio. Del total, 60\% eran hombres y $40 \%$ mujeres. La edad promedio fue de 8 años, el promedio de estancia previo al éxitus fue de 27 días, con un rango entre 1 y 204 días y una desviación estándar de 43.

Las principales causas de muerte fueron las neoplasias (26\%), las malformaciones congénitas (23\%), las inmunodeficiencias $(17 \%)$ y las causas perinatales $(11 \%)$.

De los fallecidos, solo 23\% $(n=22)$ recibió atención del equipo de cuidados paliativos. El 100\% fue acompañado por personal sanitario en el momento de la muerte y el 95\% de las familias estuvo presente.

E1 75\% $(n=71)$ de los pacientes fallecieron en la UCIP, 63\% ( $\mathrm{n}=60)$ tenía ventilación mecánica invasiva y $4 \%(\mathrm{n}=4)$ ventilación no invasiva al momento de la muerte.

Del total, 80\% $(\mathrm{n}=76)$ recibió opiáceos y el 63\% (n $=60$ ) benzodiacepinas al momento del éxitus, la mayoría en infusión continua en la UCIP porque se encontraba en ventilación mecánica al momento del fallecimiento. E1 59\% ( $\mathrm{n}=56$ ) recibió hemoderivados durante la última semana previo a la muerte, pero no con intención paliativa, todos fueron en el ámbito de la UCIP. El 74\% (n $=70)$ era portador de sonda nasogástrica y el $7 \%(\mathrm{n}=7)$ de gastrostomía.

\section{DISCUSIÓN}

El presente estudio es el primero que describe las características epidemiológicas, clínicas y evolutivas de niños con enfermedades crónicas y limitantes para la vida, que fueron susceptibles de recibir atención de cuidados paliativos en un hospital pediátrico de alta complejidad en Colombia.

Para identificar la población diana se utilizaron los códigos diagnósticos de la CIE-10 de enfermedades amenazantes y limitantes para la vida, extraídos del informe "Palliative Care Statistics for Children and Young Adults" publicado en el 2007 por el Departamento de Salud de Londres ${ }^{3}$, con el fin de comparar nuestros datos con aquellos grupos internacionales que han logrado cuantificar este tipo de pacientes y que pretende seleccionar una población que se beneficiaría de los cuidados paliativos.

La tasa de prevalencia estimada para niños y jóvenes que puedan requerir cuidados paliativos es de 10-16 por cada 10000 habitantes de entre 0-19 años (15 por cada 10000 si se excluyen las muertes neonatales). En todos los estudios, aproximadamente un 30\% padece cáncer; el
70\% restante comprende una combinación de enfermedades, principalmente neurodegenerativas, metabólicas y genéticas ${ }^{4}$. En el Hospital Pablo Tobón Uribe el 80\% de los niños susceptibles de recibir atención paliativa tenía un diagnóstico no oncológico, lo cual coincide con otros estudios de Chile ${ }^{6}$, Estados Unidos ${ }^{7}$ y Londres ${ }^{3}$.

Con respecto a las características de la población, el promedio de edad de los pacientes ( 8 años) fue similar al encontrado en el Hospital Infantil de México Federico Gómez ${ }^{8}$. Este dato difiere del observado en otro estudio realizado en México ${ }^{9}$, donde una tercera parte de los $\mathrm{pa}^{-}$ cientes era menor de 1 año, al igual que en investigaciones de España ${ }^{10}$ y Chile 6 .

Aunque la mayoría de nuestros pacientes provenían del Área Metropolitana del departamento, donde la distancia no es superior a 50 kilómetros, queda un 30\% de estos niños, que tiene que recorrer distancias más largas, algunas que superan los $500 \mathrm{~km}$, para poder ser atendidos en un hospital de alta complejidad que dé respuesta a sus múltiples necesidades. Al respecto, lo ideal es que todas las personas, independientemente de sus ingresos, del tipo de enfermedad que padezcan o de su edad, puedan tener acceso a un conjunto de servicios sanitarios básicos, incluidos los cuidados paliativos. Aunque los cuidados paliativos a cargo de especialistas son un componente de la prestación de servicios, un sistema de asistencia paliativa sostenible, de calidad adecuada y accesible debe integrarse en el contexto de la atención primaria en salud, la atención comunitaria y domiciliaria, y los proveedores de cuidados de apoyo, como familiares y voluntarios de la comunidad.

Por otro lado, entre los profesionales de la salud hay una percepción errónea respecto a que los cuidados paliativos solo se deben dar cuando se han agotado todos los esfuerzos curativos y la muerte es inminente. Los criterios de selección basados en el pronóstico, a menudo difícil de predecir en niños con enfermedades complejas distintas al cáncer, retrasan enormemente la derivación y excluyen a muchos niños de los beneficios de los cuidados paliativos pediátricos. Esto se ve reflejado en que solo el 3,7\% de nuestros pacientes fue derivado a cuidados paliativos y de estos, solo el $23 \%$ de los que fallecieron en el hospital recibieron atención por esta especialidad.

Los niños con enfermedades amenazantes y limitantes para la vida tienen necesidades complejas que requieren atención por varios especialistas, por lo que habitualmente no existe un médico o equipo tratante a su cargo. Además, se mantienen largos periodos en el hospital ( $25 \%$ permanece más de 16 días hospitalizado), sometidos a múltiples procedimientos y conductas terapéuticas, lo que hace muy difícil la toma de decisiones, sobre todo en situaciones de urgencia, ya que no existe claridad en cuanto al pronóstico ${ }^{6}$. La estancia promedio de cada 
hospitalización de estos niños encontrada en nuestra investigación fue de 11 días, lo cual es muy cercano a reportes previos $3,7,10$.

La muerte, ya no es considerada un proceso natural, se ha convertido en un "acontecimiento médico" en un entorno hospitalario que, a menudo, no está acondicionado para tratar las necesidades especiales de los niños y adolescentes que están muriendo ${ }^{4}$. Un considerable porcentaje de niños en situación terminal muere en el hospital y en entornos de cuidados de agudos, principalmente en unidades de cuidados intensivos ${ }^{11}$. Esto tiene un grave impacto en la calidad de vida y en la muerte de los niños y en la calidad de vida de las familias, lo que puede conducir a un uso incorrecto de los recursos de la asistencia sanitaria.

El hospital por su naturaleza, misión y organización no es el lugar ideal para proporcionar cuidados paliativos a niños. Se ha demostrado que los niños con enfermedades crónicas que mueren en hospitales tienen más probabilidades de haber recibido ventilación mecánica y hospitalización durante períodos más prolongados antes de su muerte ${ }^{7,12}$.

$\mathrm{Al}$ respecto, McCallum encontró en un trabajo de investigación que $83 \%$ de niños con enfermedades crónicas limitantes para la vida que fallecen en el hospital, lo hace en la unidad de cuidados intensivos y el $78 \%$ de estos pacientes se encuentra intubado al momento de la muerte. El $84 \%$ de los pacientes tenía prescrito opioides en infusión al momento del éxitus como parte del protocolo de sedación del paciente en ventilación mecánica. Estos porcentajes son muy similares a lo encontrado en nuestro estudio. Una interpretación de estos resultados es que se ofrece tratamiento excesivamente invasivo y no paliativo a los niños que se están muriendo. En efecto, "la necesidad de hacer todo lo posible para prolongar la vida del niño es una fuerza muy poderosa. Esto es característicamente evidente cuando las intervenciones curativas ya no son razonables y puede ser muy perjudicial"13.

Estudios sugieren que, donde operan las redes de cuidados paliativos pediátricos, el número de ingresos hospitalarios y la ocurrencia de muerte en el hospital de niños y adolescentes son considerablemente más bajos. Este fenómeno no solo reduce la demanda de salas de agudos y unidades de cuidados intensivos, sino que implica una mejora de la calidad de vida del paciente y de la familia 4 .

Como en otras series, el cáncer y las malformaciones congénitas fueron las primeras causas de muerte en los pacientes de este estudio ${ }^{10,14,15}$.

Es importante resaltar que todos los niños que fallecieron estuvieron acompañados por médicos y enfermeras en el momento de la muerte y solo un pequeño porcentaje (5\%) no pudo tener la compañía de sus padres. Esto contrasta mucho con el estudio de Martino, el cual reveló que los médicos estuvieron junto a su paciente en el fallecimiento en el $64 \%$ de las ocasiones y más de la mitad de los niños murió sin contacto físico con sus padres ${ }^{16}$.

En cuanto al $59 \%$ de los pacientes que recibieron hemoderivados en sus últimos días, todos ellos en el ámbito de la UCIP, es importante reflexionar acerca de que estas medidas de soporte vital no tienen un beneficio terapéutico directo. Su objetivo es restablecer funciones orgánicas, pero cuando el deterioro orgánico es irreversible, mantenerlas solo significa prolongar la agonía del enfermo. Es fundamental saber cuándo retirar y no prescribir algunos tratamientos que en los últimos días son fútiles. Hay que tener presente que hay límites éticos que no debemos sobrepasar, porque nuestras acciones serían, además de inútiles, perjudiciales. Ante un paciente en situación terminal, lo que se hace o se deja de hacer con la intención de prestarle el mejor cuidado, permitiendo la llegada de la muerte, no solamente es moralmente aceptable, sino que muchas veces llega a ser obligatorio desde la ética.

Por otro lado, el hecho de que el $81 \%$ de los pacientes que fallecieron fueran portadores de sonda nasogástrica o gastrostomía, indica el estado de fragilidad en el que se encontraban. Ahí también cabe preguntarse si en los últimos días estos dispositivos eran usados para dar alimentación enteral. Al respecto, no está indicado mantener la hidratación y la nutrición en determinadas circunstancias, fundamentalmente cuando debe administrarse por vía parenteral o mediante sonda nasogástrica u otros dispositivos como la gastrostomía, si con ello solo se va a conseguir prolongar una situación irreversible. Existen, además, posibilidades terapéuticas para controlar las hipotéticas sensaciones de hambre o de sed a las que pudiera recurrirse para no justificar su supresión. Debe brindarse el apoyo emocional adecuado, si fuera necesario, tanto para los profesionales sanitarios como para los padres que se enfrentarán a una situación de supresión terapéutica de estas características ${ }^{17-20}$.

Por último, es evidente que existe una necesidad de detectar a todos los niños que sufren enfermedades crónicas y limitantes para la vida, susceptibles de recibir atención de cuidados paliativos. Esto puede realizarse por medio de la captación activa de los mismos, con el fin de identificar a todos los pacientes que requieran este tipo de cuidados, o por el seguimiento y coordinación con los diferentes niveles asistenciales que pueden requerir este tipo de pacientes dada su alta complejidad. La incorporación de los pacientes crónicos con pronóstico de vida limitado, tanto oncológico como no oncológico, en momentos más precoces de la evolución de la enfermedad un tratamiento suministrado por un equipo de cuidados paliativos, facilitaría que pudieran recibir información completa y real del 
estado de su enfermedad, aliviar el sufrimiento evitable, favorecer la adaptación del padecimiento inevitable y tener una mejor experiencia de la enfermedad progresiva mediante la garantía de la atención, la promoción de la calidad de vida, la dignidad, la autonomía y el bienestar de los niños enfermos y sus familias.

Las conclusiones de este estudio son:

Con el fin de satisfacer las necesidades del creciente número de niños susceptibles de recibir cuidados paliativos, cada país debería recoger datos epidemiológicos acerca de la prevalencia de las enfermedades que requieren cuidados paliativos pediátricos.

Los cuidados paliativos deben desarrollarse y ser accesibles para todos los niños con enfermedades que amenazan su vida con el objetivo de mejorar el manejo de síntomas y su calidad de vida.
Mejorar la calidad de vida y la muerte en el paciente pediátrico que sufre una enfermedad incurable es un reto que debe asumirse como propio para todo el personal que labora en un hospital de alta complejidad, lo cual solo es posible con base en el conocimiento y la integración de un servicio que englobe la atención paliativa.

\section{DECLARACIÓN DE CONFLICTO DE INTERESES}

Los autores declaran no tener ningún conflicto de intereses.

\section{REFERENCIAS}

1. Organización Mundial de la Salud. Cuidados paliativos. Disponible en: http://www.who.int/ cancer/palliative/es/. Acceso el 1 de junio de 2016.

2. Departamento Administrativo Nacional de Estadística. Defunciones no fetales por grupo de edad y sexo según departamentos de ocurrencia y grupos de causas de defunción (lista Colombia 105 para la tabulación de mortalidad). Disponible en: http://www.dane.gov.co/ index.php/esp/poblacion-y-registros-vitales/nacimientos-y-defunciones/nacimientos-ydefunciones/118-demograficas/estadisticas-vitales/4675-defunciones-no-fetales-2013.

3. Cochrane $\mathrm{H}$, Liyanage $\mathrm{S}$, Nantambi R. Palliative Care Statistics for Children and Young Adults. Health and Care Partnerships Analysis. Londres: Department of Health. 2007 Mayo;1-68. Disponible en: http://webarchive.nationalarchives.gov.uk/20130107105354/http:/www.dh.gov. uk/prod_consum_dh/groups/dh_digitalassets/@dh/@en/documents/digitalasset/dh_074699.pdf

4. European Association for Palliative Care. Taskforce for Palliative Care in Children. Cuidados paliativos para lactantes, niños y jóvenes. Roma: Fundación Maruzza Lefebvre D’ovidio Onlus; 2009. Disponible en: http://www.maruzza.org/maruzza_en/Palliativecareinchildren.html

5. Departamento Administrativo Nacional de Estadística. Estimaciones de población 1985-2005 y proyecciones de población 2005-2020, nacional, departamental y municipal, por sexo y edades simples de 0 a 26 años. Disponible en: http://www.dane.gov.co/index.php/poblaciony-demografia/proyecciones-de-poblacion

6. Rosero E, Rodríguez N. Estimación de prevalencia de niños con enfermedades limitantes de la vida en un hospital pediátrico de alta complejidad. Revista de Pediatría 2013; 10(1):2-5.

7. Ananth $P$, Melvin P, Feudtner C, Wolfe J, Berry JG. Hospital use in the last year of life for children with life-threatening complex chronic conditions. Pediatrics 2015; 136(5):938-946.

8. Sosa-Sánchez K, Ramírez Mora J. Cuidados paliativos en el Hospital Infantil de México Federico Gómez: una realidad. Revista Mexicana de anestesiología. 2014; 37(3):171-176.

9. Garduño A, Hamm O, Méndez J. Experiencias en cuidados paliativos en el Instituto Nacional de Pediatría. Revista Mexicana de Pediatría 2009; 76(2):75-80.

10. Bernadá M, Methol G, González C, Giachetto G. Niños egresados de internación con condiciones de salud pasibles de cuidados paliativos: Asociación Española, 2011. Archivos de Pediatría de Uruguay 2014; 85(2):74-83.

11. Siden H. A report on location of death in pediatric palliative care between home, hospice and hospital. Paliat Med 2008; 22(7):831-834.

12. Ramnarayan P, Craig F, Petros A, Pierce C. Characteristics of death occurring in hospitalized children: changing trends. Journal of Medical Ethics, 2007; 33:255-260.

13. McCallum DE, Byrne P, Bruera E. How children die in hospital. J Pain Symptom Manage 2000; 20(6) 417-423.

14. Brandon $D$, Docherty $S$, Thorpe J. Infant and child deaths in acute care settings: Implications for palliative care. J Palliat Med 2007; 10(4):910-918.

15. Feudtner C, Hays RM, Haynes G, Geyer JR, Neff JM, Koepsell TD. Deaths attributed to pediatric complex chronic conditions: national trends and implications for supportive care services. Pediatrics 2001; 107(6):E99. 
16. Martino Alba R, Casado Flores J, Ruiz Díaz MA. Actitudes y necesidades de los intensivistas pediátricos ante la muerte de sus pacientes. Anales de Pediatría 2007; 66(4):351-356.

17. Cambra Lasaosa F. Limitación del esfuerzo terapéutico. Revista Española de Pediatría 2016; 72(1):76-79.

18. Morales VG. Limitación del esfuerzo terapéutico en cuidados intensivos pediátricos. Revista Chilena de Pediatría 2015; 86(1):56-60.

19. Valero Zanuy R, Alvarez Nido P, García Rodríguez R. ¿Se considera la hidratación y la nutrición artificial como un cuidado paliativo? Nutrición Hospitalaria 2006; 21:680-685.

20. Río MI, Shand B, Bonati P, Palma A, Maldonado A, Taboada P, et al. Hydration and nutrition at the end of life: a systematic review of emotional impact, perceptions, and decision-making among patients, family, and health care staff. Psycho-Oncology 2011; 21(9):913-921. 